\title{
The Adomian Decomposition Method for Solving a Moving Boundary Problem Arising from the Diffusion of Oxygen in Absorbing Tissue
}

\author{
Lazhar Bougoffa \\ Department of Mathematics, Faculty of Science, Al Imam Mohammad Ibn Saud Islamic University (IMSIU), \\ P.O. Box 90950, Riyadh 11623, Saudi Arabia
}

Correspondence should be addressed to Lazhar Bougoffa; bougoffa@hotmail.com

Received 15 April 2014; Revised 27 June 2014; Accepted 11 July 2014; Published 4 August 2014

Academic Editor: Ahmed Elaiw

Copyright (C) 2014 Lazhar Bougoffa. This is an open access article distributed under the Creative Commons Attribution License, which permits unrestricted use, distribution, and reproduction in any medium, provided the original work is properly cited.

\begin{abstract}
This paper begins by giving the results obtained by the Crank-Gupta method and Gupta-Banik method for the oxygen diffusion problem in absorbing tissue, and then we propose a new resolution method for this problem by the Adomian decomposition method. An approximate analytical solution is obtained, which is demonstrated to be quite accurate by comparison with the numerical and approximate solutions obtained by Crank and Gupta. The study confirms the accuracy and efficiency of the algorithm for analytic approximate solutions of this problem.
\end{abstract}

\section{Introduction}

The solution of the oxygen diffusion problem in a medium [1], which simultaneously absorbs the oxygen, consists of finding $u$ and $s$ such that

$$
\frac{\partial u}{\partial t}=\frac{\partial^{2} u}{\partial x^{2}}-1, \quad 0<x<s(t)
$$

subject to

$$
\begin{gathered}
\frac{\partial u}{\partial x}(t, 0)=0, \\
u(t, s(t))=0, \\
\frac{\partial u}{\partial x}(t, s(t))=0
\end{gathered}
$$

and the initial condition

$$
u(0, x)=\frac{1}{2}(1-x)^{2}, \quad 0<x<s(0)=1 .
$$

The above equations represent a moving boundary problem, and since not only the concentration of oxygen is always zero at the boundary but also, in addition, no oxygen diffuses across the boundary at any time, there is no relationship which contains the velocity of the moving boundary explicitly.

On comparison of this problem with the one-phase Stefan problem, we observe that the Neumann boundary condition is different to the Stefan condition, which explicitly contains the velocity of the moving boundary $[1,2]$.

Problems such as this have been treated under the name of Crank and Gupta problem and approached by numerical solutions. This is an example of a nonlinear parabolic moving boundary problem, which is difficult to get the exact solution.

Many approximate methods have been used to solve this type of problems, for example, the numerical method [29] and the method applies the Keller box finite difference scheme [10-12].

Crank, Gupta and Banik [1-3] were the first to consider integral methods applied to the oxygen diffusion problem. The integral methods have been also discussed in [13]. 
In [1], the authors proposed the following polynomial profile of fourth degree centered at $x=s$, for the resolution of (1)-(5):

$$
\begin{aligned}
u_{1}(t, x)= & \frac{s^{2}}{2}\left(1-\frac{x}{s}\right)^{2}+\left(4 u_{0}(t)-s^{2}\right)\left(1-\frac{x}{s}\right)^{3} \\
& -\left(3 u_{0}(t)-\frac{s^{2}}{2}\right)\left(1-\frac{x}{s}\right)^{4}
\end{aligned}
$$

where $u_{0}(t)=1 / 2-2 \sqrt{t / \pi}$, and then obtained

$$
\frac{d}{d t} \int_{0}^{s} u d x=-s
$$

which leads to an ODE:

$$
s^{\prime}(t)=\frac{\left(20+8 u_{0}^{\prime}(t)\right) s}{8 u_{0}(t)+s^{2}} \quad \text { with } t \geq \frac{4}{25 \pi} .
$$

A similar analysis has been applied in [2] for the following polynomial profile of fourth degree centered at $x=0$ :

$$
\begin{aligned}
u_{2}(t, x)= & u_{0}+\frac{1}{2}\left(s^{2}-12 u_{0}\right)\left(\frac{x}{s}\right)^{2} \\
& +\left(8 u_{0}-s^{2}\right)\left(\frac{x}{s}\right)^{3}+\frac{1}{2}\left(s^{2}-6 u_{0}\right)\left(\frac{x}{s}\right)^{4},
\end{aligned}
$$

where $u_{0}(t)=u(t, 0)$ and $s$ is determined from

$$
\frac{d}{d t} \int_{0}^{s} x u d x=u_{0}(t)-\frac{1}{2} s^{2}
$$

The resulting ODEs are given by

$$
\frac{d u_{0}}{d t}=-\frac{5 s^{4}+30 s^{2} u_{0}+24 u_{0}^{2}}{s^{2}\left(5 s^{2}+24 u_{0}\right)}, \quad \frac{d s}{d t}=-\frac{60\left(s^{2}-4 u_{0}\right)}{s\left(5 s^{2}+24 u_{0}\right)}
$$

The purpose of this paper is to apply the Adomian decomposition method [14-37] to find the solution of (1), (3), and (4), that is, the oxygen diffusion $u(t, x)$, and then obtain an expression for the location of the moving boundary, which gives an ODE to solve for $s(t)$ as a function of time. In addition, we will show that the partial solution in the $t$ directions requires less computational work by using the initial condition only. Also, using an a priori estimate, we prove the uniqueness of the solution of (1)-(5).

\section{Analysis of the Method}

Consider the general problem:

$$
\frac{\partial u}{\partial t}=\frac{\partial^{2} u}{\partial x^{2}}-g(x), \quad 0<x<s(t)
$$

which is the governing equation, subject to the boundary condition

$$
\frac{\partial u}{\partial x}(t, 0)=h(t)
$$

the Dirichlet boundary condition

$$
u(t, s(t))=p(t),
$$

the Neumann boundary condition

$$
\frac{\partial u}{\partial x}(t, s(t))=q(t)
$$

and the initial condition

$$
u(0, x)=\varphi(x), \quad 0<x<s(0)=1 .
$$

Our problem contains, as a special case, the above system which describes the oxygen diffusion problem.

Based on the Adomian decomposition method, we write (12) in Adomian's operator-theoretic notation as

$$
L_{x x} u=\frac{\partial u}{\partial t}+g(x)
$$

where

$$
L_{x x}=\frac{\partial^{2}}{\partial x^{2}}
$$

Applying the inverse linear operator $L_{x x}^{-1}=\int_{x}^{s(t)} \int_{x}^{s(t)}(\cdot) d x d x$ to (17) and taking into account that $u(t, s(t))=p(t)$ and $(\partial u / \partial x)(t, s)=q(t)$, we obtain

$$
\begin{aligned}
u(t, x)= & p(t)-q(t)(s-x) \\
& +\int_{x}^{s(t)} \int_{x}^{s(t)} g(x) d x d x+\int_{x}^{s(t)} \int_{x}^{s(t)} \frac{\partial u}{\partial t} d x d x .
\end{aligned}
$$

Define the solution $u(t, x)$ by an infinite series of components in the form

$$
u(t, x)=\sum_{n=0}^{\infty} u_{n}(t, x)
$$

Consequently, the components $u_{n}$ can be elegantly determined by setting the recursion scheme:

$$
\begin{aligned}
u_{0} & =p(t)-q(t)(s-x)+\int_{x}^{s(t)} \int_{x}^{s(t)} g(x) d x d x, \\
u_{n+1} & =\int_{x}^{s(t)} \int_{x}^{s(t)} \frac{\partial u_{n}}{\partial t} d x d x, \quad n \geq 0,
\end{aligned}
$$

for the complete determination of these components.

Replace $p(t)=q(t)=0$ and $g(x)=1$ into the recursion scheme (21) to get

$$
\begin{aligned}
& u_{0}=\frac{1}{2 !}(s-x)^{2}, \\
& u_{1}=\frac{s^{\prime}}{3 !}(s-x)^{3}, \\
& u_{2}=\frac{s^{\prime 2}}{4 !}(s-x)^{4}+\frac{s^{\prime \prime}}{5 !}(s-x)^{5},
\end{aligned}
$$


A polynomial profile of fifth degree is now obtained by the Adomian decomposition method, which is the truncated decomposition series $u(t, x)=u_{0}(t, x)+u_{1}(t, x)+u_{2}(t, x)$. So that

$$
u(t, x)=\frac{1}{2 !}(s-x)^{2}+\frac{s^{\prime}}{3 !}(s-x)^{3}+\frac{s^{\prime 2}}{4 !}(s-x)^{4}+\frac{s^{\prime \prime}}{5 !}(s-x)^{5}
$$

and which automatically satisfies the boundary conditions (3) and (4).

We can now obtain an expression for the location of the moving boundary, $s(t)$. This is derived from integrating (12) with respect to $x$ from 0 to $x$ and taking into account that $(\partial u / \partial x)(t, 0)=h(t)$; we obtain

$$
\frac{\partial u}{\partial x}=h(t)+\int_{0}^{x} g(x) d x+\int_{0}^{x} \frac{\partial u}{\partial t} d x .
$$

Substitute $x=s$ into (24) and using the fact that $(\partial u / \partial x)(t, s)=q(t)$. Thus

$$
\int_{0}^{s(t)} g(x) d x+\int_{0}^{s(t)} \frac{\partial u}{\partial t} d x=q(t)-h(t), \quad s(0)=1 .
$$

Using the following Leibniz's rule for differentiation under the integral sign:

$$
\frac{d}{d t} \int_{0}^{s(t)} u(t, x) d x=\int_{0}^{s(t)} \frac{\partial u}{\partial t} d x+u(t, s(t)) \frac{d s}{d t},
$$

and taking into account that $u(t, s(t))=p(t)$, we obtain

$$
\int_{0}^{s(t)} \frac{\partial u}{\partial t} d x=\frac{d}{d t} \int_{0}^{s(t)} u(t, x) d x-p(t) \frac{d s}{d t} .
$$

Substituting (27) into (25), we get

$$
\int_{0}^{s(t)} g(x) d x+\frac{d}{d t} \int_{0}^{s(t)} u(t, x) d x-p(t) \frac{d s}{d t}=q(t)-h(t),
$$

where $s(0)=1$. If we consider $p(t)=q(t)=h(t)=0$ and $g(x)=1$, then (28) becomes

$$
\frac{d}{d t} \int_{0}^{s(t)} u(t, x) d x=-s
$$

Substitute the profile equation (23) into (29) gives an ODE to solve for $s(t)$, namely,

$$
\frac{s^{2} s^{\prime}}{2 !}+\frac{s^{3} s^{\prime 2}}{3 !}+\frac{s^{4} s^{\prime \prime}}{4 !}+\frac{s^{4} s^{\prime 3}}{4 !}+3 \frac{s^{5} s^{\prime} s^{\prime \prime}}{5 !}+\frac{s^{6} s^{\prime \prime \prime}}{6 !}=-s
$$

with $s(0)=1$. So that

$$
\frac{s s^{\prime}}{2 !}+\frac{s^{2} s^{\prime 2}}{3 !}+\frac{s^{3} s^{\prime \prime}}{4 !}+\frac{s^{3} s^{\prime 3}}{4 !}+3 \frac{s^{4} s^{\prime} s^{\prime \prime}}{5 !}+\frac{s^{5} s^{\prime \prime \prime}}{6 !}+1=0
$$

We now can determine the location of the moving boundary $s(t)$ as a function of time by solving the nonlinear equation
(31). Indeed, the solution $s(t)$ follows immediately by setting the following form:

$$
s=\sqrt{1+2 \lambda t}
$$

where $\lambda \in \mathfrak{R}$ is a parameter to be determined. Simple computations lead to

$$
\begin{gathered}
s s^{\prime}=\lambda, \quad s^{\prime \prime} s^{3}=-\lambda^{2}, \\
s^{\prime \prime \prime} s^{5}=3 \lambda^{3}, \quad s^{\prime \prime} s^{\prime} s^{4}=-\lambda^{3} .
\end{gathered}
$$

Substituting these expressions into (31), we obtain

$$
\frac{\lambda}{2 !}+\frac{\lambda^{2}}{3 !}-\frac{\lambda^{2}}{4 !}+\frac{\lambda^{3}}{4 !}-3 \frac{\lambda^{3}}{5 !}+3 \frac{\lambda^{3}}{6 !}+1=0
$$

or equivalently,

$$
\lambda^{3}+6 \lambda^{2}+24 \lambda+48=0
$$

Consequently, we find $\lambda=-3.192143275966643 \approx-3.2$, which is a real root of this equation.

Hence, the concentration and the location of the moving boundary for $0 \leq t<1 / 6.4 \approx 0.156$ can be represented fairly accurately by the approximate expression equation (23) and

$$
s=\sqrt{1-6.4 t}
$$

respectively.

It should be noted that this solution is applicable for the time $0 \leq t<1 / 6.4$ only.

Graphs have been drawn to show the concentration distributions and the positions of the moving boundary at various times $0 \leq t<0.156$ (Figures 1 and 2).

As it was mentioned in [13], we see that the method of Gupta and Banik [2] only starts at $t=t^{*}=4 / 25 \pi$, with $s\left(t^{*}\right)=1$, and is applicable for the time interval $4 / 25 \pi \leq$ $t \leq \pi / 16$. For $t \geq 4 / 25 \pi$, Laplace solutions give analytical solutions for the short time problem [1]. Also, the Gupta and Banik method [2] requires that $s^{2}-4 u_{0} \geq 0$. Since $s(0)=1$ it follows that $u_{0}(0) \leq 0.25$. This is incompatible with the initial condition at $t=0$ and so this method can only start at $t=t^{*}>0$, with the assumption that $s\left(t^{*}\right)=0$.

Table 1 shows that the values obtained by using the Adomian decomposition method, which are in a very good agreement with those calculated by Gupta and Banik, for small times.

An expression for surface concentration can be obtained by putting $x=0$ in (23). Thus

$$
u(t, 0)=\frac{1}{2 !} s^{2}+\frac{s^{\prime}}{3 !} s^{3}+\frac{s^{\prime 2}}{4 !} s^{4}+\frac{s^{\prime \prime}}{5 !} s^{5}
$$

which can be compared with the numerical solutions [1]

$$
u_{1}(t, 0)=\frac{1}{2}-2 \sqrt{\frac{t}{\pi}} .
$$

Comparative figures are given in Table 2. 
TABLE 1: A comparison for the position of the moving boundary as obtained from (a) numerical evaluation by using Runge-Kutta method, (b) approximation solution [1], (c) numerical method [1], and (d) ADM.

\begin{tabular}{lcccc}
\hline Values of $x$ & Numerical solution & Approximate solution & Numerical method & ADM \\
\hline 0 & - & - & 1 & 0.9967 \\
0.051 & 1 & 0.9996 & 0.9922 & 0.82073 \\
0.060 & 0.9974 & 0.9817 & 0.9719 & 0.7848 \\
0.080 & 0.9750 & 0.9393 & 0.9352 & 0.6985 \\
0.100 & 0.9321 & & & 0.6000 \\
\hline
\end{tabular}

TABLE 2: A comparison for the surface concentration $u(t, 0)$ with $u_{1}(t, 0)$ [1].

\begin{tabular}{lcccc}
\hline Values of $x$ & Analytical & Numerical & Approximate & ADM \\
\hline 0.04 & 0.274328 & 0.274496 & 0.274324 & 0.229152 \\
0.08 & 0.180852 & 0.180969 & 0.180846 & 0.150304 \\
0.12 & 0.109134 & 0.109228 & 0.109118 & 0.0714560 \\
0.15 & 0.048771 & 0.048893 & 0.048648 & 0.0123200 \\
\hline
\end{tabular}

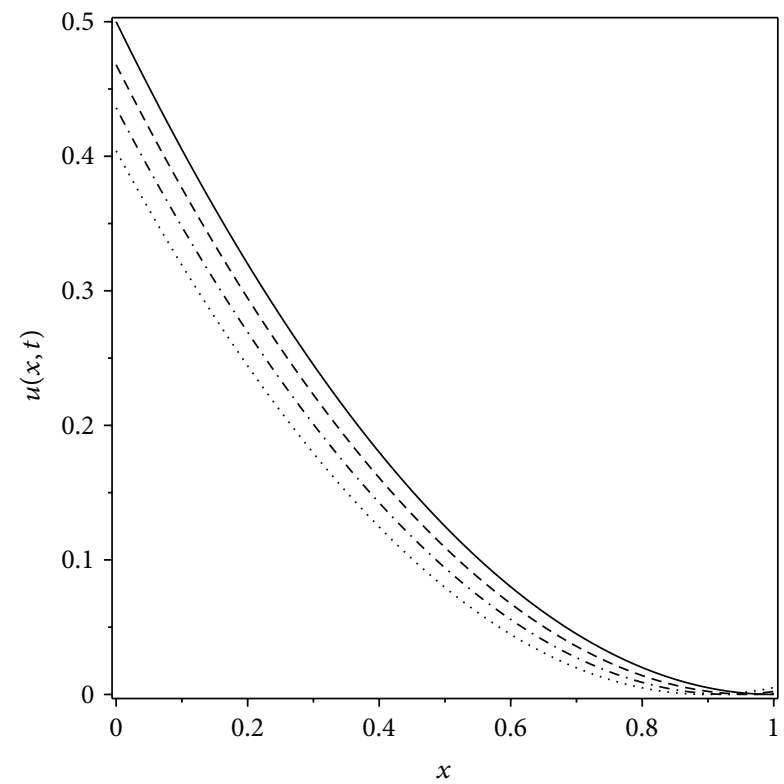

FIGURE 1: Concentration distributions $u(x, t)$ for different values of $t$. Solid line: $t=0$, dashed line: $t=0.01$, dash dotted line: $t=0.02$, and dotted line: $t=0.03$.

An important note can be made here that the $t$-solution can be obtained by using the initial condition equation (16) only. To do this, we apply the inverse linear operator $L_{t}^{-1}(\cdot)=$ $\int_{0}^{t}(\cdot) d t$ to both sides of (12) and use the initial condition equation (16) to obtain

$$
u(t, x)=\varphi(x)-g(x) t+\int_{0}^{t} \frac{\partial^{2} u}{\partial x^{2}} d t
$$

where $\varphi(x)=(1 / 2)(1-x)^{2}$ and $g(x)=1$. So that the decomposition method consists of decomposing the

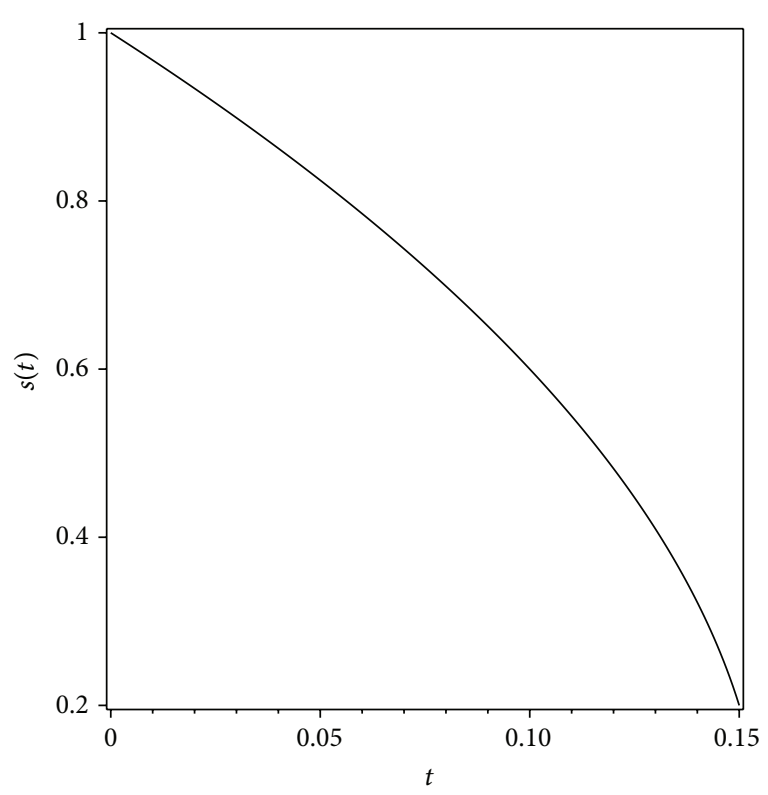

FIGURE 2: Variation of the moving boundary position equation (33) with the time $t$ for $0 \leq t<0.156$.

unknown function $u(t, x)$ into a sum of components defined by the series $u(t, x)=\sum_{n=0}^{\infty} u_{n}(t, x)$. Thus the components can be elegantly determined in a recursive manner as will be discussed later; we therefore set the recurrence scheme:

$$
\begin{aligned}
u_{0} & =\frac{1}{2}(1-x)^{2}-t, \\
u_{n+1} & =\int_{0}^{t} \frac{\partial^{2} u_{n}}{\partial x^{2}} d t, \quad n \geq 0 .
\end{aligned}
$$


In view of this, the components $u_{0}(t, x), u_{1}(t, x), u_{2}(t, x), \ldots$ are immediately determined as

$$
\begin{aligned}
u_{0} & =\frac{1}{2}(1-x)^{2}-t, \\
u_{1} & =t \\
u_{n+1} & =\int_{0}^{t} \frac{\partial^{2} u_{n}}{\partial x^{2}} d t=0, \quad n \geq 1 .
\end{aligned}
$$

Consequently, the $t$-solution is readily found to be

$$
u(t, x)=\frac{1}{2}(1-x)^{2},
$$

which is a very good approximation and the same approximate solution obtained upon using the Laplace transforms when it has been assumed that the boundary has not moved from its original position, $s=1[1]$.

To obtain $s(t)$ as a function of time, substituting the profile equation (42) into (29), we get

$$
\frac{d s}{d t}=-2 \frac{s}{(1-s)^{2}}, \quad s(0)=1,
$$

which leads to the implicit solution

$$
\ln s-2 s+\frac{s^{2}}{2}=-2 t-\frac{3}{2}
$$

Graph has been drawn to show the solution for the moving boundary $s(t)$ in Figure 3.

\section{A Priori Estimate}

Here we establish an a priori estimate which ensures the uniqueness of the solution of the given free boundary value problem.

Proposition 1. For any solution $u(t, x)$ of (12) that satisfies (2)-(5) there exists a positive constant $C$ independent on $u(t, x)$ such that

$$
\begin{aligned}
& \sup _{0 \leq \tau \leq T}\left[\int_{0}^{s(\tau)} u^{2}(\tau, x) d t d x+\int_{0}^{\tau} \int_{0}^{s(\tau)}\left(\frac{\partial u}{\partial x}\right)^{2} d t d x\right] \\
& \quad \leq C\left(\int_{0}^{1} g^{2}(x) d x+\int_{0}^{1} \varphi^{2}(x) d x\right),
\end{aligned}
$$

where $g \in L_{2}[0, s]$.

Proof. Multiply both sides of (12) by $u$, integrating over $\Omega^{\tau}=$ $[0, \tau] \times[0, s(\tau)]$. After applying integration by parts and taking into account that $u(t, s)=0,(\partial u / \partial x)(t, s)=0$ and $(\partial u / \partial x)(t, 0)=0$, we obtain

$$
\begin{aligned}
& \frac{1}{2} \int_{0}^{s(\tau)} u^{2}(\tau, x) d t d x-\frac{1}{2} \int_{0}^{s(\tau)} \varphi^{2}(x) d x \\
& \quad+\int_{0}^{\tau} \int_{0}^{s(\tau)}\left(\frac{\partial u}{\partial x}\right)^{2} d t d x=-\int_{0}^{\tau} \int_{0}^{s(\tau)} g(x) u(t, x) d t d x
\end{aligned}
$$

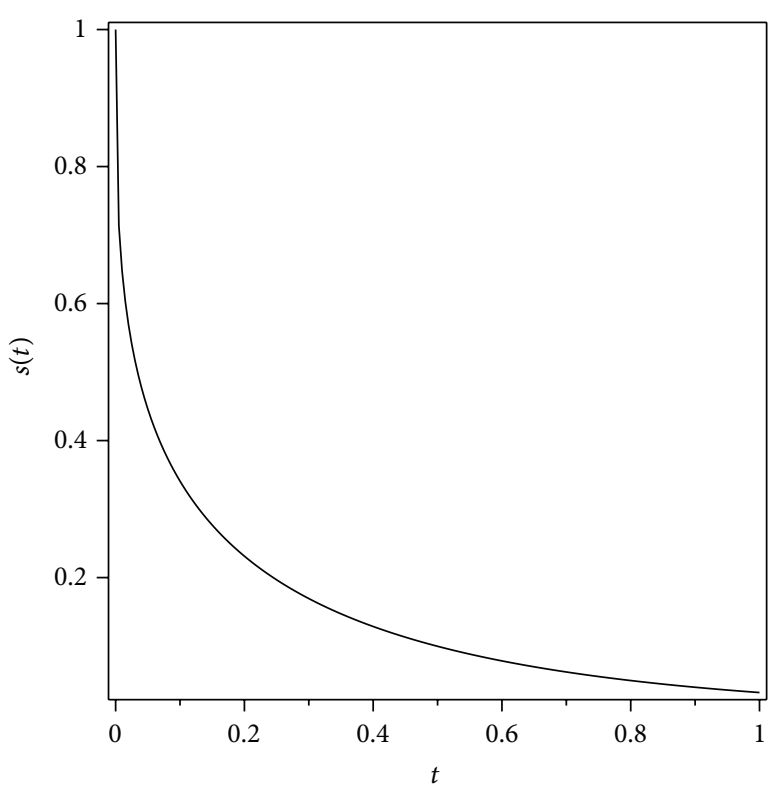

FIGURE 3: Moving boundary position $s(t)$ in terms of $t$ obtained from (44).

Use the $\epsilon$-inequality: $2|a b| \leq(1 / \epsilon) a^{2}+\epsilon b^{2}, \epsilon>0$ to estimate the term which arises in the right-hand side of (46).

Thus

$$
\begin{aligned}
& \int_{0}^{s(\tau)} u^{2}(\tau, x) d t d x+2 \int_{0}^{\tau} \int_{0}^{s(\tau)}\left(\frac{\partial u}{\partial x}\right)^{2} d t d x \\
& \leq \leq \frac{1}{\epsilon} \int_{0}^{\tau} \int_{0}^{s(\tau)} g^{2}(x) d x+\epsilon \int_{0}^{\tau} \int_{0}^{s(\tau)} u^{2}(t, x) d t d x \\
& \quad+\int_{0}^{s(\tau)} \varphi^{2}(x) d x
\end{aligned}
$$

Since $\Omega^{\tau}=[0, \tau] \times[0, s(\tau)] \subset[0, T] \times[0,1]$, it follows

$$
\begin{gathered}
\int_{0}^{s(\tau)} u^{2}(\tau, x) d t d x+\int_{0}^{\tau} \int_{0}^{s(\tau)}\left(\frac{\partial u}{\partial x}\right)^{2} d t d x \\
\leq T \int_{0}^{1} g^{2}(x) d x+\int_{0}^{1} \varphi^{2}(x) d x \\
+\epsilon \int_{0}^{\tau} \int_{0}^{s(\tau)} u^{2}(t, x) d t d x .
\end{gathered}
$$

Using Gronwall's lemma, we obtain

$$
\begin{gathered}
\int_{0}^{s(\tau)} u^{2}(\tau, x) d t d x+\int_{0}^{\tau} \int_{0}^{s(\tau)}\left(\frac{\partial u}{\partial x}\right)^{2} d t d x \\
\leq C\left(\int_{0}^{1} g^{2}(x) d x+\int_{0}^{1} \varphi^{2}(x) d x\right) .
\end{gathered}
$$

Now, replacing the right-hand side of (49) by its upper bound with respect $\tau$ in the interval $[0, T]$, we obtain the desired inequality. 


\section{Conclusion}

In this work we investigated the moving boundary problem arising from the diffusion of oxygen in absorbing tissue. The approximate method obtained upon using the ADM would specially be useful to calculate the concentration and the position of the moving boundary at an arbitrary time. Graphs have been drawn to show the concentration-distributions and the progress of the moving boundary with respect to time at various times. The work confirmed the power of the Adomian method in handling this example of a nonlinear parabolic moving boundary problem, without an exact solution.

\section{Conflict of Interests}

The author declares that there is no conflict of interests regarding the publication of this paper.

\section{References}

[1] J. Crank and R. S. Gupta, "A moving boundary problem arising from the diffusion of oxygen in absorbing tissue," Journal of the Institute of Mathematics and Its Applications, vol. 10, pp. 19-33, 1972.

[2] R. S. Gupta and N. C. Banik, "Approximate method for the oxygen diffusion problem," International Journal of Heat and Mass Transfer, vol. 32, no. 4, pp. 781-783, 1989.

[3] R. S. Gupta and N. C. Banik, "Constrained integral method for solving moving boundary problems," Computer Methods in Applied Mechanics and Engineering, vol. 67, no. 2, pp. 211-221, 1988.

[4] R. S. Gupta and D. Kumar, "Complete numerical solution of the oxygen diffusion problem involving a moving boundary," Computer Methods in Applied Mechanics and Engineering, vol. 29, no. 2, pp. 233-239, 1981.

[5] R. S. Gupta and A. Kumar, "Variable time-step method with coordinate transformation," Computer Methods in Applied Mechanics and Engineering, vol. 44, no. 1, pp. 91-103, 1984.

[6] S. G. Ahmed, "Approximate method for oxygen diffusion in a sphere with simultaneous absorption," International Journal of Numerical Methods for Heat and Fluid Flow, vol. 9, no. 6, pp. 631-642, 1999.

[7] S. G. Ahmed, "A numerical method for oxygen diffusion and absorption in a sike cell," Applied Mathematics and Computation, vol. 173, no. 1, pp. 668-682, 2006.

[8] S. Çatal, "Numerical approximation for the oxygen diffusion problem," Applied Mathematics and Computation, vol. 145, no. 2-3, pp. 361-369, 2003.

[9] V. Gülkaç, "Comparative study between two numerical methods for oxygen diffusion problem," Communications in Numerical Methods in Engineering, vol. 25, no. 8, pp. 855-863, 2009.

[10] S. L. Mitchell and M. Vynnycky, "Finite-difference methods with increased accuracy and correct initialization for onedimensional Stefan problems," Applied Mathematics and Computation, vol. 215, no. 4, pp. 1609-1621, 2009.

[11] S. L. Mitchell and M. Vynnycky, "An accurate finite-difference method for ablation-type Stefan problems," Journal of Computational and Applied Mathematics, vol. 236, no. 17, pp. 4181-4192, 2012.

[12] S. L. Mitchell, M. Vynnycky, I. G. Gusev, and S. S. Sazhin, "An accurate numerical solution for the transient heating of an evaporating spherical droplet," Applied Mathematics and Computation, vol. 217, no. 22, pp. 9219-9233, 2011.

[13] S. L. Mitchell, "An accurate application of the integral method applied to the diffusion of oxygen in absorbing tissue," Applied Mathematical Modelling, 2014.

[14] G. Adomian, Nonlinear Stochastic Operator Equations, Academic Press, Orlando, Fla, USA, 1986.

[15] G. Adomian, Nonlinear Stochastic Systems Theory and Applications to Physics, Kluwer Academic Publishers, Dordrecht, The Netherlands, 1989.

[16] G. Adomian, Solving Frontier Problems of Physics: The Decomposition Method, Kluwer Academic, Dordrecht, The Netherlands, 1994.

[17] G. Adomian and R. Rach, "Transformation of series," Applied Mathematics Letters, vol. 4, no. 4, pp. 69-71, 1991.

[18] R. Rach, G. Adomian, and R. E. Meyers, "A modified decomposition," Computers and Mathematics with Applications, vol. 23, no. 1, pp. 17-23, 1992.

[19] G. Adomian and R. Rach, "Inhomogeneous nonlinear partial differential equations with variable coefficients," Applied Mathematics Letters, vol. 5, no. 2, pp. 11-12, 1992.

[20] G. Adomian and R. Rach, "Nonlinear transformation of seriespart II," Computers and Mathematics with Applications, vol. 23, no. 10, pp. 79-83, 1992.

[21] G. Adomian and R. Rach, "Modified decomposition solution of nonlinear partial differential equations," Applied Mathematics Letters, vol. 5, no. 6, pp. 29-30, 1992.

[22] G. Adomian and R. Rach, "Modified decomposition solution of linear and nonlinear boundary-value problems," Nonlinear Analysis: Theory, Methods \& Applications, vol. 23, no. 5, pp. 615619, 1994.

[23] G. Adomian and R. Rach, "Analytic solution of nonlinear boundary value problems in several dimensions by decomposition," Journal of Mathematical Analysis and Applications, vol. 174, no. 1, pp. 118-137, 1993.

[24] G. Adomian, "Modification of the decomposition approach to the heat equation," Journal of Mathematical Analysis and Applications, vol. 124, no. 1, pp. 290-291, 1987.

[25] G. Adomian, "A new approach to the heat equation-an application of the decomposition method," Journal of Mathematical Analysis and Applications, vol. 113, no. 1, pp. 202-209, 1986.

[26] A. Wazwaz, Partial Differential Equations and Solitary Waves Theory, Higher Education Press, Beijing, China, 2009.

[27] A. M. Wazwaz, "Equality of partial solutions in the decomposition method for partial differential equations," International Journal of Computer Mathematics, vol. 65, no. 3-4, pp. 293-308, 1997.

[28] A. Wazwaz, "A reliable modification of Adomian decomposition method," Applied Mathematics and Computation, vol. 102, no. 1, pp. 77-86, 1999.

[29] J. Duan and R. Rach, "A new modification of the Adomian decomposition method for solving boundary value problems for higher order nonlinear differential equations," Applied Mathematics and Computation, vol. 218, no. 8, pp. 4090-4118, 2011.

[30] J. Duan, R. Rach, A. Wazwaz, T. Chaolu, and Z. Wang, "A new modified Adomian decomposition method and its multistage form for solving nonlinear boundary value problems with Robin boundary conditions," Applied Mathematical Modelling, vol. 37, no. 20-21, pp. 8687-8708, 2013. 
[31] R. Rach, "A convenient computational form for the Adomian polynomials," Journal of Mathematical Analysis and Applications, vol. 102, no. 2, pp. 415-419, 1984.

[32] J. Duan, "Convenient analytic recurrence algorithms for the Adomian polynomials," Applied Mathematics and Computation, vol. 217, no. 13, pp. 6337-6348, 2011.

[33] A. Wazwaz, "A new algorithm for calculating Adomian polynomials for nonlinear operators," Applied Mathematics and Computation, vol. 111, no. 1, pp. 53-69, 2000.

[34] L. Bougoffa, R. C. Rach, and A. Mennouni, "An approximate method for solving a class of weakly-singular Volterra integrodifferential equations," Applied Mathematics and Computation, vol. 217, no. 22, pp. 8907-8913, 2011.

[35] L. Bougoffa, R. C. Rach, and A. Mennouni, "A convenient technique for solving linear and nonlinear Abel integral equations by the Adomian decomposition method," Applied Mathematics and Computation, vol. 218, no. 5, pp. 1785-1793, 2011.

[36] L. Bougoffa and R. C. Rach, "Solving nonlocal initial-boundary value problems for linear and nonlinear parabolic and hyperbolic partial differential equations by the Adomian decomposition method," Applied Mathematics and Computation, vol. 225, pp. 50-61, 2013.

[37] L. Bougoffa, R. C. Rach, and A. Wazwaz, "Solving nonlocal initial-boundary value problems for the Lotka-von Foerster model," Applied Mathematics and Computation, vol. 225, pp. 715,2013 . 


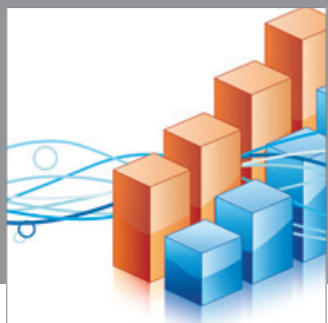

Advances in

Operations Research

mansans

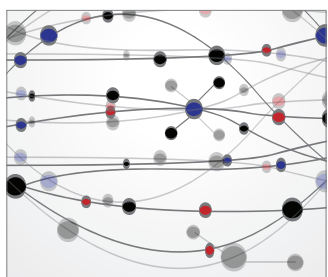

The Scientific World Journal
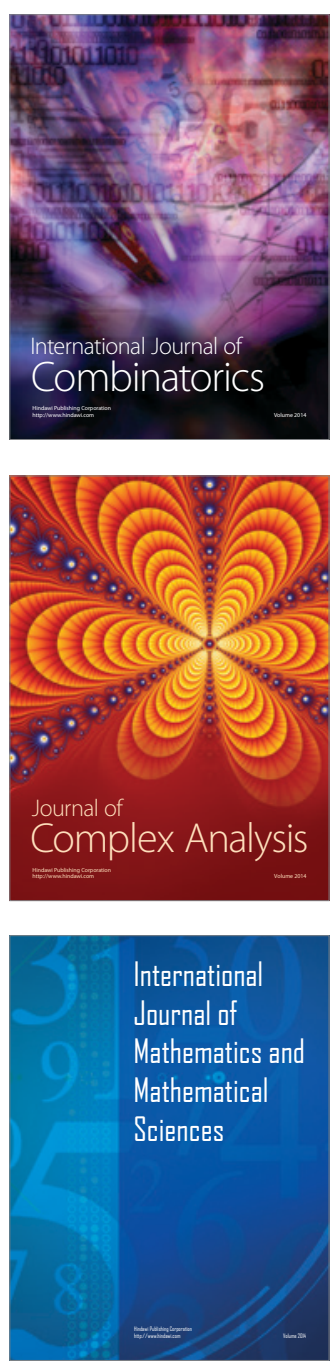
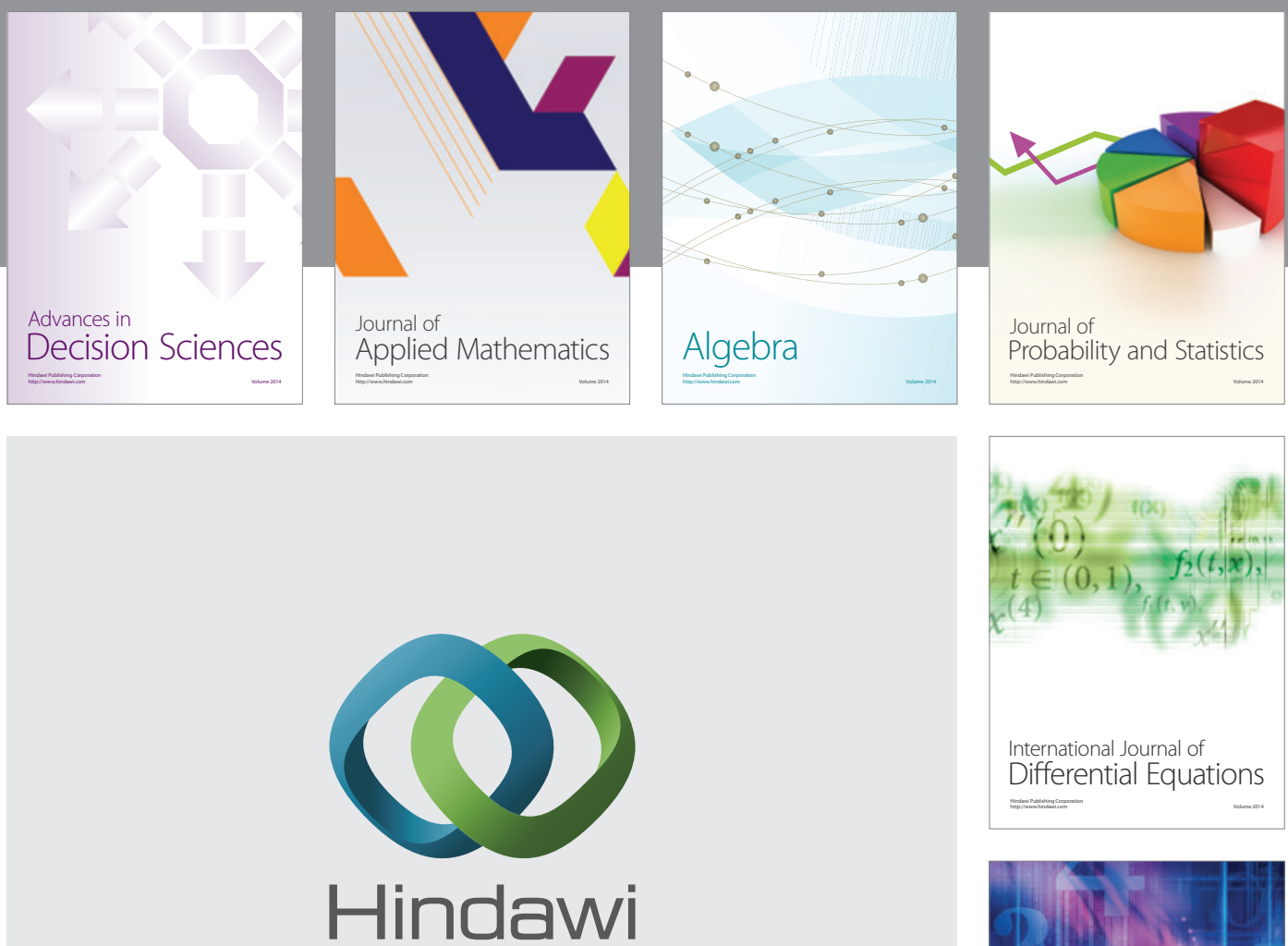

Submit your manuscripts at http://www.hindawi.com
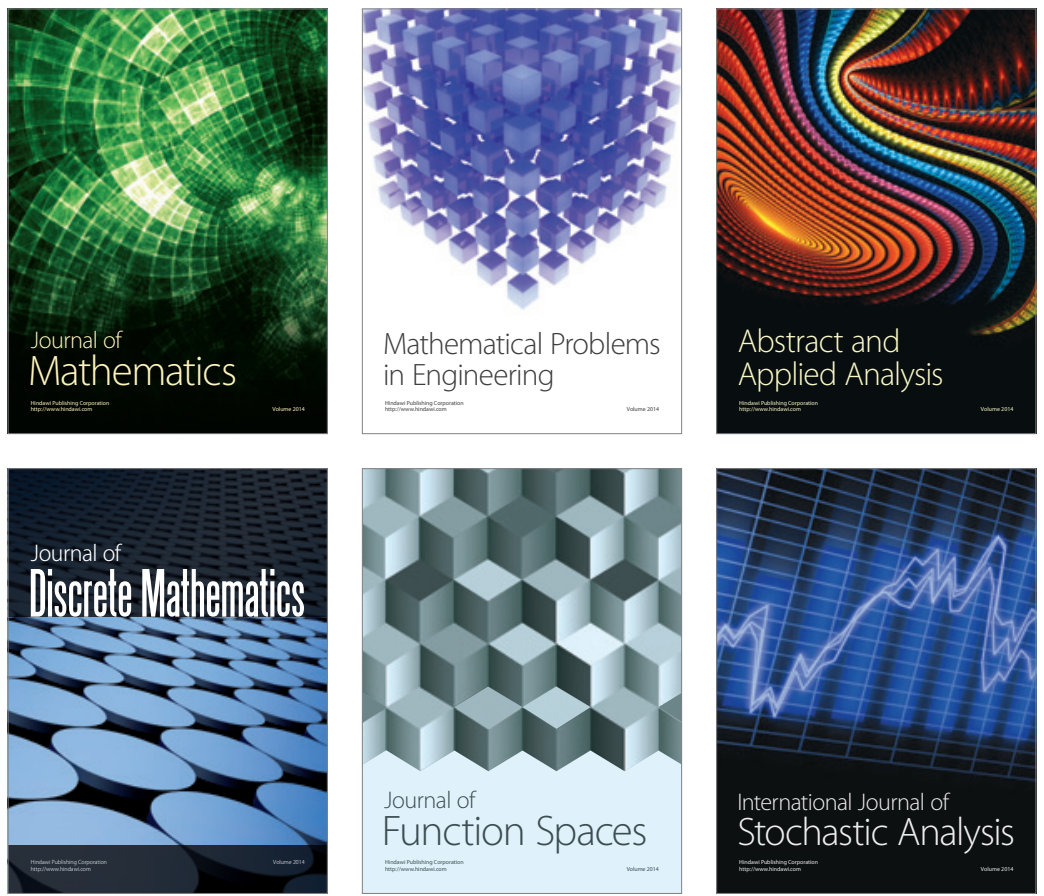

Journal of

Function Spaces

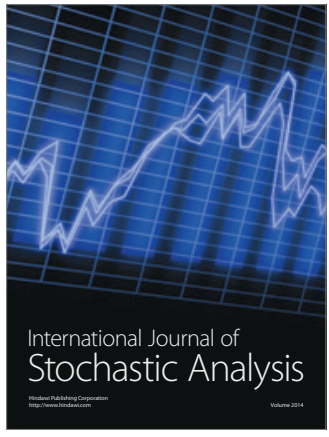

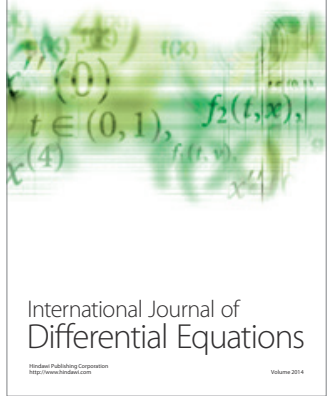
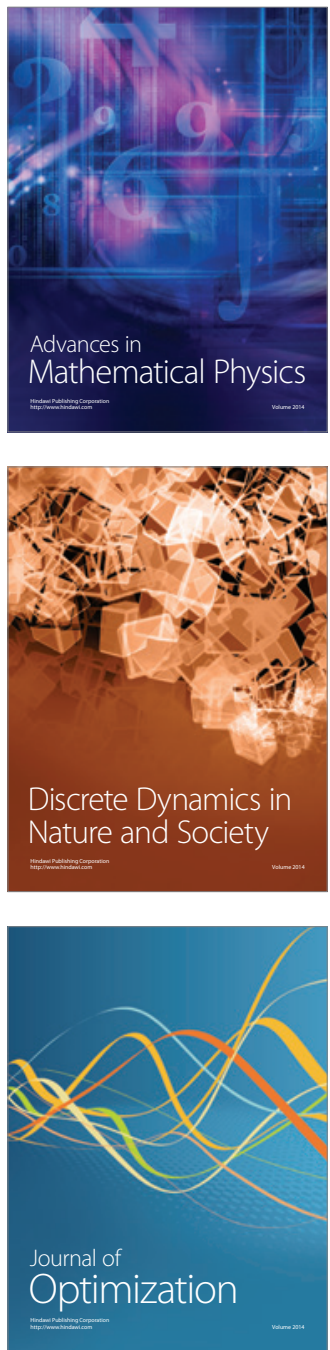